\title{
A vigilância da qualidade da água para consumo humano - desafios e perspectivas para o Sistema Único de Saúde
}

\author{
Surveillance in drinking-water quality - challenges \\ and perspectives for the Brazilian Health System
}

Ma rcelo Bessa Freitas 1

Carlos Machado de Freitas 2

\footnotetext{
1 Laboratório de Educação Profissional de Vigilância em Saúde, Escola Politécnica de Saúde Joa quim Venâncio, Fiocruz. Av. Brasil 4.365,

Manguinhos, 21045-900, Rio de Jan ei ro RJ. bessa@fiocruz.br

2 Cen tro de Estu dos em Saúde do Trabalhador e Ecologia Humana, Escola Naci onal de Saúde Pública Sergio Arouca, Fiocruz
}

Resumo O controle da qualidade da água de consumo humano se torn ou uma ação de saúde pública a partir da década de 1970, quando a portaria No 52 Bsb 77 do Mnistério da Saúde insti tuiu a norma de potabilidade em todo o território nacional. Entret a n to, a impl em entação de um programa de vigilância da qualidade da água só oco rreu a partir da criação do Sistema Nacional de Vigilância Ambiental em Saúde em 1999, e da publicação da portaria 1.469 em 2000. O objetivo deste arti go é discutir os desafios que se colocam pa ra a vigilância da qualidade da água, não só como proposta racionalizadora das ações do Estado, mas como prática sanitária de vigilância em saúde que tenha como perspectiva incorporar a promoção e a proteção da saúde ao conjun to de medidas adotadas pelo SUS, a tendendo ainda às seguintes diretrizes: geração de dados, análise e disseminação da informação; descentralização; intersetorialidade e participação da sociedade. $O$ artigo está organizado de modo a descrever, a partir de uma perspectiva histórica, o processo de normatização e regulação da qualidade da água pa ra o consumo humano, abordando os principais desafios e perspectivas da vigilância da qualidade da água no sentido da busca por ações intersetoriais e descen tralizadas, a bertas a uma gestão participa tiva voltada para o controle social.

Palavras-chave Água para consumo humano, Vigilância em saúde, Controle social 


\section{Introdução}

A partir do final dos anos 80 e início dos anos 90, a definição de vigilância em saúde pública se difunde internacionalmen tecomo a sistemática coleta, análise e interpretação dos dados acerca de even tos de saúde específicos que afetam a população, estando integrada com a rápida disseminação dos dados para todos aqueles que são res ponsáveis pela prevenção e controle (Thacker et al., 1996; Waldman, 1998). A vigilância em saúde pública se insere em um amplo processo de reformulação das práticas de saúde pública nos anos 80 e 90, convertendo-se em elemen to informacional estratégi co que, possibilitou, em um con tex to de con s trangimentos fiscais, subsidiar tomadas de decisões, avaliar a relação custo-efetividade dos programas de intervenção e estabel ecer pri oridades na alocação dos parcos recursos financeiros governamentais nas políticas de saúde (Th acker et al., 1996; Sh erman\&Ma thi a s, 1996; Wigle \& Mowat, 1999).

Neste mesmo período, o Brasil iniciava seu processo de democratização da socied ade, com forte atuação do movimen to sanitário, materializados no marco institucional do con ceito ampliado de saúde na Constituição de 1988, assim como a lei no 8.080 de 1990. Esta lei, significou a institucionalização da vigilância em uma perspectiva mais abrangente, ainda que manten do separadas a vigilância sanitária da epidemiológica. Para a vi gilância sanitária a lei 8.080 forn eceu maior abrangência às ações, incluin do en tre as suas com petências a vigilância de produtos, serviços, dos ambi en tes e dos processos de trabalho, através da execução direta ou mediante a participação de outros setores. Além disso, tem a função de coordenar a Rede Nacional de Laboratório para a Qualidade em Saúde. Para a vigilância epidemiológica, a lei atribui-lhe as responsabilidades pelas ações vo ltadas para a detecção e prevenção de qualquer mudança nos fatores determinantes e condicionantes e para o con trole de doenças (não se limitandosom en te às transmissívés) ou agravos (Costa \& Rozenfeld, 2000).

A Constituição de 1988 e a lei 8.080 con tribuíram para que na década de 1990 se intensificassemas discussões em torno da reorganização do sistema de vigilância epidemiológica na perspectiva do SUS, tornan do possíve se conceber a proposta de ação baseada na vigilância em saúde, que incorporaria a identificação e d ivulgação de fatores condicionantes - modo de vida (condições e estilos de vida) - e determinantes socioambientais dos problemas de saúde, articulando-os com o con ju n to de políticas econômicas e sociais visando ao con trole de causas e à redução do ris co da doença e outros agravos (IESUS, 1993). Entretanto, estas discussões não deixaram de ser influenciadas pelas novas propostas que surgiram no final dos anos 90 nos países industrializados, como as de vigilância ambiental em saúde pública (Thacker et al., 1996) ou de abord a gem holística para a vigilância em saúde pública (Levy, 1996). Estas propostas, que se originaram nos países industrializados, con tinham pelo men os três elemen tos que deveriam estar integrados: 1) a vigilância de efeitos sobre a saúde, como agravos e doenças, $t$ a refa tradicionalmen te realizada pela vigilância epidemiológica; 2) a vigilância de perigos, como agen tes químicos, físicos e bioló gi cos que possam oca si onar doenças e agravos, ta refa tradicionalmente realizada pela vigilância sanitária; 3 ) a vigilância de exposições, através do moni toramento da exposição de indivíduos ou grupos populacionais a um a gente ambi ental ou seus efei tos dinicamente ainda não aparen tes (subdínicos ou pré-díni$\cos )$, desafio para a estrutu ração da vigilância ambiental.

Influenciado por estas propostas e com financiamen to do Ba n co Mundial, a partir do final dos anos 90, a través do proj eto de Estruturação do Sistema Nacional de Vigilância em Sa ú de (Vigisus), se inicia a estrutu ração e institucionalização da vi gilância ambiental no âmbi to do Mnistério da Saúde. Em maio de 2000 é publ i c ado o dec reto no 3.450, que estabel eceu no Centro Nacional de Epidemiologia (Cenepi), a gestão do Sis tema Nacional de Vigilância Ambiental em Saúde (Si nvas). No entanto em 7 de março de 2005, com a publicação em Diário Oficial da instrução normativa SVS № 1, esse sistema foi redefinido como Subsistema de Vigilância em Sa ú de Ambi ental (SNVSA).

Um dos com ponentes do SNVSA é o setor de vi gilância da qualidade da água para con sumo humano, ten do como ... finalidade o mapeamen to de áreas de risco em determinado território (...), para avaliação das características de pota bilidade (...), com vistas a asseg u rar a qualidade da água e evitar que as pessoas adoeçam pela presença de patógenos ou contaminantes presentes nas coleções hídricas (Fundação Nacional de Saúde, 2002). Este setor é também o responsável pela coordenação do Sistema de Informação de Vi gilância e Con trole da Quali- 
dade da Água de Con sumo Humano (Sisagua) e pela identificação, acompanhamento e avaliação das ações e as metas da Programação Pactuada In tegrada de Epidemiologia e Controle de Doenças (PPI-ECD) correspondentes a sua competência. As informações que vêm alimentando o banco de dados dizem respeito aos aspectos físico-químicos, químicos e microbiológi cos e dados sobre a qualidade, a vazão, a população abastecida e a localização do sistema de abastecim en tode água.

Esse modelo de vigilância se aproxima mu ito mais das propostas racionalizadoras de vigilância em saúde su r gidas nos países industriali$z$ ados, s en do cen trada na vigilância dos agen te $s$ (o que inclui produtos) e da exposição, do que das propostas de vi gilância da saúde orientada para a identificação e divulgação de fatores condicionantes - modo de vida (condições e estilos de vida) - e determinan tes socioambientais dos probl emas de saúde em populações.

O objetivo deste artigo é discutir os desafios que se apresentam para a vigilância da qu alidade da água para con sumo humano dentro da vigilância em saúde ambi en tal, não só como proposta racionalizadora das ações do Estado, mas também como prática sanitária de vigilância em saúde, ten do a perspectiva de incorporar a promoção e a proteção da saúde ao conju n tode medidas adotadas pelo SUS, que atenda ainda às seguintes diretrizes: 1) geração de dados, análise e disseminação da informação; 2) descentralização; 3 ) intersetorialidade e 4) participação da soci ed ade.

Pa ra realizar tal obj etivo, descreve-se, a partir de uma pers pectiva histórica, o processo de n orm a tização e regulação da qualidade da água pa ra o con sumo humano, no con tex to intern acional (item 1) e brasilei ro (item 2). Em segui$\mathrm{da}$ (item 3) abordam-se os principais desafios e perspectivas da vigilância da qualidade da água, no sentido da busca por ações intersetoriais e descentralizadas, abertas a uma gestão participativa volt ada para o con trole social.

\section{A normatização da qualidade da água para consumo humano}

A qualidade da água se tornou uma questão de interesse para a saúde pública no final do século 19 e início do século 20. An teriormente, a qualidade era associada apenas a aspectos estéticos e sensoriais, tais como a cor, o go s to e o odor. Mé todos para melh orar o aspecto estéti- co e sen sorial da água já foram en con trados há 4.000 anos a.C. em documen tos escritos em sânscri to. Entretanto, na Grécia antiga utilizavam-se técnicas como a filtração, a exposição ao sol e a fervu ra para mel horar aqualidade da água. Mesmo que motivados mais pela aparência turva que a água apresentava, os gregos apontavam empiricamente para a existência de relações causais en tre água e enfermidades, como fez Hipócrates (Usepa, 1999).

Na metade do século 19, ocorrem avanços na compreensão da relação entre água contaminada e doenças, destacando-se o trabalho do epidemiologista John Sn ow, que, em 1855, provou que um surto de cólera em Lon d res estava associado a poços de abastecimento público contaminados por esgoto. Mais tarde, em 1880, Louis Paster demonstrou pela Teoria dos Germes como organismos microscópicos (micróbios) poderiam transmitir doenças por meio da água. Nessa mesma época, cien tistas de s cobri ram que a turbi dez não estava somen te rel acionada a aspectos estéticos. O material particulado em água poderia con ter organismos patogênicos e material fecal.

No início do século 20, em função dessas de s cobertas científicas, muitos sistemas de tratamen to de água são construídos nos Estados Unidos, em pregan do a filtração lenta como estra té giade con trole da qua lid ade da água. Anos mais tarde, naquele mesmo país, a cloração é empregada pela pri m ei ra vez no Estado de New Jersey em 1908. O utros desinfetantes também são utilizados nesse mesmo período, como o ozônio na Eu ropa. As iniciativas de potabilização da água de con sumo humano se deram antes do estabelecimen to de padrões e normas de qualidade. Algumas iniciativas pion ei ras merecem destaque. Em 1914, uma norma federal americana, el a borada pelo serviço de saúde pública da época, estabelecia um padrão para qualidade microbi oló gica da água. Porém, essa norma se aplicava somen te à água produzida por sistema de abastecimento e transportada via navios e trens para outros Estados, e se limitava a contaminantes capazes de causar doenças contagiosas (Usepa, 1999).

Os valores máximos permiti dos ou os limites máximos de contaminação (MCLs) foram sendo estabelecidos em função de estudos e bioensaios toxicológicos, e de componentes químicos e físico-químicos capazes de alterar as propriedades organolépticas ou sensoriais da água, que, além de causar rejeição de con sumo, podem esti mular condições propícias à di- 
minuição da vida útil de toda a aparelhagem hidráulica com con seqü en tes prejuízos econômicos.

$\mathrm{Na}$ atualidade, a Organização Mundial de Saú de (OMS) é a instituição que acompanha e recomenda os valores máximos permitidos, a partir dos estu dos toxicológi cos realizados em todo o mundo e publicados em diferen tes revistas e even tos científicos especializados no tema. Alguns países, como os Estados Unidos, o Canadá, e a Comunidade Eu ropéia, apesar de se basearem também nas recomendações da OMS, estimulam pesquisas toxicológicas e bi oensaios que, reci procamente, acabam servindo de referência tanto para a OMS como para os demais países. Todas as normas de potabilidade no Brasil seguem basicamente os padrões recomendados pela Organização Mundial de Saú de no Guidelines for Drinking-Water Quality (WHO, 1996).

A partir de estu dos e levantamen tos da situação da maioria dos sistemas de abastecim ento de água, que apresentavam uma qualidade fora dos padrões de potabilidade, no início dos anos 70 é criada nos Estados Uni dos uma norma nacional de potabilidade de água para consumo humano, a Safe Drinking Water Act, de 1974, que estabelecia um padrão nacional de qualidade da água. Por esta lei, toda água captada e tratada pelas companhias de abastecimen to daquele país deveria corresponder a este padrão de potabilidade, ou seja, apresentar s eus constituin tes orgânicos, inorgânicos e microbiológicos den tro dos valores máximos permitidos, ou seja, a água utilizada para fins de con sumo humano (beber, lavar, cozinhar e para fins higiênicos) não deveria ser capaz de provocar nen hum risco ou dano à saúde humana. Atualmente a Safe Drinking Water Act está subm etida ao órgão de controle ambiental americano, o Un i ted States Environ m ental Protecti on Agen cy (Usepa).

$\mathrm{Na}$ Eu ropa, a atual norma de qualidade de água para fins de consumo humano tem abrangência em todos os países da comunidade européia. A Drinking Water Directive (DWD) 98/83/EC é submetida a cada cinco anos a um processo de revisão, que tem como principal obj etivo definir estra tégias rel a tivas à gestão do s is tema de produção de água potável, no sentido de propor a inclusão de novos parâmetros químicos ou biológicos, a revisão dos limites máximos de contaminação dos parâmetros já exis ten tes e a discussão sobre tendências de gerenciam en tode ri s cos para o setor (EC, 2003).
As normas de potabilidade têm se apres entado como um instrumental técnico-jurídico elaborado pelas autoridades sanitárias, com o a poio de instituições técnico-científicas, a ser cumprido pelos órgãos de fiscalização e vigilância do setor saúde e pelas em presas públicas e privadas de abastecimen to de água. Essas normas constituem-se como referências técnicas que imprimem um padrão de qualidade ao "produto" água, seja ele obti do diretamen te da natureza (sem tratamen to ou água bruta) ou por meio de processos químicos em plantas de tratamento. Entretanto, a simples existência de n ormas reguladoras não asseg u ra a certificação e a manutenção de padrões de qualidade para os domínios sanitário e econômico. Esse conjun to de valores normativos e o seu cumprimento como lei precisam ser continuamen te discutidos por toda a socied ade civil, pelos ge stores públ i cos e o meio científico, a fim de que se assegure uma maior amplitude e legitimidade do processo.

\section{A normatização da qualidade da água no Brasil}

No Brasil, a normatização da qualidade da á g u a pa ra con sumo humano é iniciada na década de 1970. A primeira norma de potabilidade foi cri ada no Brasil pelo dec reto federal no 79.367 de 9 de março de 1977, que estabel eceu a competência doMinistério da Sa ú de sobre a def in ição do padrão de potabilidade da água para consumo humano, a ser observado em todo território nacional, a través da portaria no $56 \mathrm{Bsb}$, publicada em 14 de março de 1977.

Considera-se esta a primeira norma de potabilidade, uma vez que abrangia diferentes constituintes químicos e microbiológi cos po tencialmen te patog ên icos à saúde humana. En tretanto, é import a n te regi s trar que em 24 de maio de 1974 é publicada no Di á rio Oficial a lei federal no 6.050 que dispunha sobre a necessidade de flu oretação da água de sistemas de abastecimen to proven i en tes de estação de tratamento.

A 56 Bsb aprovou a norma e o padrão de potabilidade da água para fins de con sumo humano. De acordo com este dec reto, caberia às Sec retarias de Saú de dos Estados e equivalen te aos territórios e Distrito Federal o exercício de fiscalização e o con trole do ex a to cumprimento das Normas e do Padrão de Potabilidade.

Segundo Formaggia et al. (1996), em 1986, o Mnistério da Saúde, através da extinta Divi- 
são de Ecologia Humana e Saúde Ambiental, efetuou um levantamento das atividades exercidas pelas Secret a rias Estaduais de Saúde(SES), com relação ao que passou a se chamar de Vigilância Sanitária da Qualidade da Água para Consumo Humano. Na época, constatou-se que, à exceção do Paraná, os demais Estados não exerciam nen huma ativid ade relacion ada à vigilância da qualidade da água, ou, se exerciam, não o faziam de forma sistemática e planejada. O Ministério da Saúde decidiu então c riar em 1986 o Programa Naci onal de Vigilância da Qualidade da Água para Con sumo Humano, o qual, entre outras metas, pretendia prestar auxílio técnico e financei ro às Secretarias Estaduais de Saú de para que estas: iniciassem um programa de vigilância da qualidade da água; efetiva s s ema revisão da legislação; capacitassem tecnicamen te os profissionais das SESs para atua rem em vigilância da qualidade da água; e definissem estratégias em conjunto com as SESs para garantir apoio laboratorial nece s sá rio à verificação do cumprim en toda legislação quanto ao padrão físico-químico e $\mathrm{b}$ acteriológicoda água.

A primeira revisão da portaria no $56 \mathrm{Bsb} / 77$ foi iniciada em 1988, e apesar de estar inicialm en te restrita à participação de setores governam entais de saúde e de companhias estaduais de abastecimen to de água e órgãos estaduais de con trole ambiental, consegu iu po steri orm en te envo lver as vi gilâncias sanitárias, os Laboratórios de Sa ú de Pública (LACENs), a comunidade científica e algumas associações de dasse, na discussão da revisão, que forn eceu subsídios à n ova portaria, a $36 \mathrm{GM}$, publicada em 19 de ja$\mathrm{n}$ ei ro de 1990. En tre a principais inovações introduzidas pela $36 \mathrm{GM} / 90$, estão: 1) a definição de con trole e vigilância da qualidade; 2) a definição de serviço e sistema de abastecimen to de água; e 3) a inclusão e revisão de alguns parâmetros químicos e microbiológicos.

A segunda revisão ocorreu dez anos depois, extrapolando a recomendação do pra zo máximo de cinco anos, conti dos na $36 \mathrm{GM} / 90$, após a sua promulgação. A revisão da $36 \mathrm{GM} / 90$ foi con duzida pelo Mnistério da Saúde, por meio da Coordenação Geral de Vigilância Ambi en tal em Saúde (CGVAM), em parceria com o Departamen to de Engenharia de Saúde Pública (DENSP), da Fundação Naci onal de Saúde (Funasa) e com a representação da Organização Pan-Am ericana de Saúde (OPAS) e da Organização Mundial de Saúde (OMS), no Brasil (MS, 2000). Nessa última revisão, ampliou-se mais o processo participativo, na con fecção da norma, uma vez que houve a colaboração de diversas en tidades dos setores públ i co, privado e de organizações não-governamentais. Além disso, a própria Funasa coordenou uma con sulta pública, para receber contribuições de profissionais, entidades e instituiçõos de várias regiões do país. Em 29 de de zem bro de 2000, foi publ icada a portaria no 1.469 (MS, 2000), estabel ecen do o con trole e a vigilância da qualidade da água para o con sumo humano e seu padrão de potabilidade. O prazo de adequação das instituições ou órgãos para o cumprimento da Norma venceu em janeiro de 2003 , exceto para o monitora m en to de cianob act é ri as, que teve um prazo-limite de implementação de três anos após a publicação da portaria.

A principal inovação trazida pela portaria 1.469 foi a classificação dos tipos de sistemas de abastecimento de água em: sistema coletivo, que se constitui de uma instalação composta por um conjunto de obras civis, materiais e equipamentos, destinados à produção e à distribuição canalizada de água potável para as populações, sob a responsabilidade do poder público, mesmo que administrada em regime de con cessão ou permissão; e sistema ou solução alternativa de abastecimen to de água - que se constitui de toda modalidade de abastecimen to coletivo de água, distinta do sistema coletivo, incluindo, por exemplo, fontes, poços comunitários, distribuição por veículo transport ador, instalações con dominiais horizontal e vertical.

Em função dessa classificação, a portaria 1.469 estabeleceu deveres e responsabilidades aos órgãos de saúde, encarregados de realizar a vigilância, e às em presas de abastecimen to, administradas sob o domínio públ i co ou privado, responsáveis pelo controle da qualidade da água. Ambos deven do monitorar os parâmetros de qualidade, de s deas áreas de pro teção do corpo hídri co utilizado para a captação, até o tratamen to e distribuição. Esta última revisão também incorporou um novo parâmetro microbiológico, a pesquisa de cianobactérias e cianotoxinas. Além de recom endações a re s peito da operacionalidade para a remoção de cistos de Giárdia spp e oocistos de Cryptosporidium sp., a partir da melhora da eficiência no processo de filtração.

O quadro 1 apresenta algumas diferenças en tre as duas normas de potabilidade que já estiveram em vi gor no país e a norma atual. Enquanto as duas primeiras normas, a Bsb 56 e a 
Quadro 1

Comparativo das portarias de Potabilidade de Água para o Con sumo Humano de 1977 a 2000.

\begin{tabular}{|c|c|c|c|}
\hline Normas/Características & $\begin{array}{l}\text { Portaria no } 56 \text { BSB } \\
\text { de } 13 / 03 / 1977\end{array}$ & $\begin{array}{l}\text { Portaria no } 36 / 90 \\
19 / 01 / 1990\end{array}$ & $\begin{array}{l}\text { Portaria no } 1.469 / 00 \\
02 / 01 / 2001\end{array}$ \\
\hline Amostra gem e freqüência & $\begin{array}{l}\text { Apres enta uma metodologia } \\
\text { de amostra gem e freqüência, } \\
\text { baseada no número de } \\
\text { habitantes e no tipo de } \\
\text { constituintea ser analisado. }\end{array}$ & $\begin{array}{l}\text { Apre senta uma metodologia } \\
\text { de amostra gem em freqüência, } \\
\text { baseada no número de } \\
\text { habitantes e no tipo de } \\
\text { constituinte que vai ser } \\
\text { analisado. }\end{array}$ & $\begin{array}{l}\text { Inclui a necessidade de um plano } \\
\text { de amostra gem e frequiência } \\
\text { para os sistemas alternativos } \\
\text { de abastecimento de água. }\end{array}$ \\
\hline Valor máximo permitido & $\begin{array}{l}\text { Inclui val ores máximos } \\
\text { permiti dos e valores máximos } \\
\text { desejáveis. }\end{array}$ & $\begin{array}{l}\text { Reproduz os valores máximos } \\
\text { permiti dos recom endados pela } \\
\text { OMS, Comunidade Eu ropéia } \\
\text { e Norma Can adense. } \\
\text { Não apre senta mais a definição } \\
\text { de valores máximos desejáveis. }\end{array}$ & $\begin{array}{l}\text { A revisão dispõe sobre novos } \\
\text { constituintes, tais como } \\
\text { cianotoxinas, cianob actérias, } \\
\text { e recom endações operaci onais } \\
\text { sobre criptosporidium, seguindo } \\
\text { basicamen te os valores máximos } \\
\text { recom endados pela OMS. }\end{array}$ \\
\hline Ações de scen tralizadas & $\begin{array}{l}\text { As ações de fiscalização e } \\
\text { con trole são exercidas pelo MS } \\
\text { em articulação com as SESs. }\end{array}$ & $\begin{array}{l}\text { As ações de fiscalização e } \\
\text { con trole são exercidas pelo MS } \\
\text { em articulação com as SESs. }\end{array}$ & $\begin{array}{l}\text { Repassa uma maior responsabilidade } \\
\text { das ações de vi gilância para } \\
\text { os municípios. }\end{array}$ \\
\hline Ações interinstitucionais & Não previa. & Não previa. & $\begin{array}{l}\text { Si tua a importância dos órgãos } \\
\text { de con trole ambi en tal, no con trole } \\
\text { da qualidade da água da bacia } \\
\text { hidrográfica usada para captação. }\end{array}$ \\
\hline In formação & $\begin{array}{l}\text { As informações são } \\
\text { centralizadas pelo MS } \\
\text { em articulação com as SESs. } \\
\text { Não prevê a disponibilidade } \\
\text { das informações sobre a } \\
\text { qualidade da água. }\end{array}$ & $\begin{array}{l}\text { As informações são } \\
\text { centralizadas pelo MS } \\
\text { em articulação com as SESs. } \\
\text { Não prevê a disponibilidade } \\
\text { das informações sobre a } \\
\text { qualidade da água. }\end{array}$ & $\begin{array}{l}\text { Destaca que os respon sá veis pelos } \\
\text { sistemas de abastecim en to devem } \\
\text { repassar as informações sobre } \\
\text { a qualidade da água ao con s elho } \\
\text { de defesa do con sumidor, com } \\
\text { periodicidade mínima anual } \\
\text { e com periodicidade mensal às } \\
\text { a utoridades de saúde pública. }\end{array}$ \\
\hline Con trole e vigilância & $\begin{array}{l}\text { Não define vigilância, mas as } \\
\text { SESs se obri gam a manter } \\
\text { um registro permanente de } \\
\text { in formações sobre a qualid ade } \\
\text { da água dos sistemas de } \\
\text { abastecimen to públ i co, } \\
\text { bem como forn ecer ao MS, } \\
\text { notificandoimediatamente } \\
\text { a ocorrência de fato } \\
\text { epidemiológi co que possa } \\
\text { estar relacion ado ao } \\
\text { comprometimen to da } \\
\text { qualidade da água fornecida. }\end{array}$ & $\begin{array}{l}\text { Define con trole e vigilância } \\
\text { da qualidade da água. Define } \\
\text { as responsabilidades e } \\
\text { competências sobre a vigilância } \\
\text { (MS e SESs) e con trole (os } \\
\text { serviços de abastecimento } \\
\text { de água). }\end{array}$ & $\begin{array}{l}\text { Define con trole e vi gilância da } \\
\text { qualidade da água sobre os } \\
\text { sis temas de abastecimento de água } \\
\text { coletivo e alternativo. Define as } \\
\text { responsabilidades e competências } \\
\text { sobre a vi gilância e con trole, e } \\
\text { sobre os dois ti pos de sistemas de } \\
\text { abastecimento, no sentido de } \\
\text { operar estes sistemas de acordo } \\
\text { com as Normas da ABNT (1996). }\end{array}$ \\
\hline
\end{tabular}


36/90, traziam basicamente valores máximos permiti dos e indicações estatísticas para realização de amostra gem e freqüência, a 1.469 incorporou por meio de uma discussão mais ampla os diferentes sistemas de abastecimento (coletivo e alternativo) e a atribuição precisa de responsabilidade operacional por estes sistemas, sob a pers pectiva dos ri s cos à saúde públ ica associados à vulnerabilidade do manancial, às práticas operacionais do tratamen to, e integridade física da rede de distribuição. Em 25 de março de 2004, a portaria no 1.469 foi revogada e substituída pela portaria no 518 , que reprodu z iu intei ramente o con teú do da 1.469.

\section{A vigilância da qualidade da água: desafios e perspectivas}

Apesar de possuir uma norma de potabilidade desde 1977, a vigilância da qualidade da água para consumo humano só foi implementada no Brasil como um programa, a partir da criação do Si s tema Nac i onal de Vigilância Am bi ental em Saú de (Funasa, 2002). Es te programa se en contra estruturado como um subsistema, e tem como uma de suas responsabilidades a coordenação de um sistema de informação de vigilância e controle da qualidade da água de consumo humano (Sisagua). As informações que vêm alimentando o banco de dados dizem respei to aos aspectos físico-químicos, químicos e microbiológi cos e dados sobre a qualidade, a vazão, a população abastecida e a localização do sistema.

O Sisagua vem sendo alimentado com informações geradas pelos responsáveis pela operação dos sistemas de abastecimento de água (dados de monitoramen to microbiológico, químico e físico-químico obtidos no controle da qualidade da água) e responsáveis pela vigilância da qualidade da água, no caso, as secretarias Estaduais e Municipais de Saúde.

O diagnóstico obtido a partir da vigilância, teoricamente, possibilita aos gestores tom a rem as decisões em torno dos sistemas de abastecimen to coletivos e altern a tivo s, no sen ti do de se exigirem as intervenções adequ ad a s, qu a n do há ocorrência de não-con form i d ades com a qualidade da água. Este diagnósti co também pode permitir o mapeamen to de áreas ou sistemas de maior vulnerabilidade ambiental e técnica, ajud an doa definir as que são prioritárias.

Entretantó a existência de alguns entraves impossibilita que a vigilância da qualidade da água seja efetivam en te exercida na pers pectiva da proposta de ação baseada no modelo de vigilância em saúde. Como apontado na introdução, esse modelo incorporaria a identificação e divulgação de fatores condicionantes modo de vida (condições e estilos de vida) - e determinantes socioambientais dos problemas de saúde em populações, articulando-se com um conjunto de políticas econômicas e sociais visando ao controle de causas e a redução do risco da doença e outros agravos. Dentre esses entraves destacamos: 1) a geração de dados, análise e disseminação da inform a ção;2) a descentralização das ações; 3 ) a inters etorialidade nas ações; 4) a participação da sociedade para o con trole social.

Em relação à geração de dados, análise e disseminação da inform a ção, devemos lem brar que, nas discussões e propostas do modelo de vigilância em saúde no início dos anos 90 , a coleta de dados era uma ativid ade que envo lvia os três níveis de governo (federal, estadual e municipal), de modo a assegurar a elaboração de indicadores, assim como a consolidação e análise dos mesmos de forma a permitir o acompanhamen to das condições de saúde da pop ulação. O âmbito estadual, por exemplo, s eria responsável por manter o fluxo adequado das informações que permitissem o acompanhamen to das condições de saúde da população. Entretanto, o que se verifica na atualidade é completamen te distin to. Em um levantamento recen te realizado por Bezerra et al. (2004), em 2003, verificou-se que 78\% dos Estados e o Distrito Federal alimentam o banco de dados do Sisagua com informações sobre os sistemas de abastecimento, que, por sua vez, recebem informações dos municípios.

En tret an to, somen te 19\% dos Estados realizam avaliações sistemáticas da vi gilância da qualidade da água, visando à redução da morbimortalid adedas doenças de veiculação hídrica. Neste mesmo levantamento, foi possível concluir que a portaria no 518 se en contra parcialmente implantada nas unidades da federa çã o, necessitando que haja um maior em penho em todos os níveis de governo para que se efetivem as ações da vi gilância da qualid adeda água para consumo. $\mathrm{E}$ ainda que os dados gerados e analisados re sultem em informações que permitam o acom $p$ a$n$ h a m en to das condições de saúde da pop u la ção e o desenvolvimen to de estraté gias de redução da morbimortalidade e promoção da saúde.

Além do probl ema da geração e análise dos dados, converten do-os em informações, é po s- 
sível verificar que essa não-disponibilidade de informações é um problema que atinge diferen tes níveis do poder públ i co. Por exemplo, as pá ginas de Intern et doMin istério da Sa ú de não permitem o acesso às informações sobre o $\mathrm{Si}$ sagua, mesmo parcialmente, o que acaba contribuindo para limitar o controle social, pilar do SUS. Essa disponibilidade de informações está prevista na lei no 8.080/90, a qual refere que a divulgação de informações em saúde é atri buição da União, Estados, Distri to Federal e Municípios. Além disso, a portaria no 518, no arti go 7으, parágrafo VI, estabelece como competência do nível municipal ga rantir à população informações sobre a qualidade da água e ri s coà saúde associados.

Esta não-disponibilidade de informações, de um tema de amplo interesse nacional, contraria uma das atribuições do nível federal, na proposta do modelo de vigilância em saúde, que é o de divul gar informações visandoà ampliação da consciência sanitária e à participação da população nas atividades de vigilância e controle de agravos. Nesse senti do, ONGs como o Instituto de Defesa do Consumidor (Idec) têm sido mais ativas na inves ti gação e disponibilização de informações que perm item iden tificar que Estados como SantaCatarina, Paraná, Rio de Janei ro e São Paulo e suas respectivas capitais só cumprem parcialmente a portaria no 1.469 (Folha de S. Paulo, 9/4/2004, C1, p. 1).

Em relação à descentralização, na perspectiva da proposta do modelo de vigilância em saúde, cabe à vigilância con formar, organizar, produzir e difundir informações que respondam às exigências de consolidação do SUS e da proposição da gestão descentralizada, com ênfase na municipalização. No que con cerne à vigilância da qualidade da água, isto implica que seu processo deve responder por um amplo repasse de responsabilidade para o nível local (município), o que envo lve aadministração e a operação e manutenção do sistema, assim como a vigilância, a informação e o controle da qualid ade da água.

No Brasil, a descentralização das ações do sistema de saúde vem sendo encaminhada de forma a aten der aos preceitos da lei orgânica no 8.080/90 e da NOB SUS 01/96, que repassa amplos poderes de execução de ações de saúde aos municípios. Pode-se afirmar também que, apesar da falta de estrutura ainda existen te em gran de parte dos municípios brasileiros para a execução destas ações, o governo federal, em con ju n to com os governos estaduais, vem dan- do algum suporte organizacional e estrutural aos municípios para que efetivem tal con trole, principalmente, a partir da reestruturação da capacidade dos Laboratórios de Saúde Pública de Referências (LACENs) e da realização de capacitações e treinamentos de técnicos e gestores dos Estados e municípios. Além disso, o repasse de recursos via PPI-ECD aos municípios tem propiciado o apoio financei ro e material p a ra a execução das ações de con trole e prevenção de doenças, agenda que inclui a qualidade da água, definida como um fator de ri s coà saúde humana. De qualquer modo, ainda há muito para se avançar, diante da falta de estrutura, de recu rsos e de trabalhadores qualificados que as Secretarias Estaduais e Municipais de Sa úde al egam não possuir.

No que se refere à intersetorialização das ações e à inclusão do processo participativo numa gestão integrada da água como fator de ris co à saúde humana, os desafios se apresentam em diferen tes mom en tos e situações. Conforme aponta Jo u ravi ev (2001), as águas nece ssitam de uma gestão integrada, envolven do o manejo dos recu rsos hídri cos em seus diferentes usos, o que inclui a água para o consumo humano, a segurança alimentar e a proteção de ecossistemas. Deste modo, não é possívd separar as ações de vigilância da qualidade da água pa ra con sumo humano das ações inters etoriais que visem transformar o atual quadro de degradação dos recursos hídricos que afetam a saúde humana (Moraes \& Jordão, 2002).

Em relação à inters etorialização das ações, pode-se afirmar que há uma dificuldade histórica de se planejar, de s envolver e implementar ações en tre os diferen tes setores. As raízes dessa dificuldade se infiltram por obstáculos do âmbitopo lí tico, sociocultural, organizac i onal e jurídico, tendo em vista que os setores, instituições e seus arc a bouços legais foram e ainda têm sido constituídos de forma especializada, através de códigos (linguagens) próprios e herméti cos (MS, 2003).

A falta de políticas e ações intersetoriais e in tegradas voltadas para a qualidade da água pode ser exemplificado com o recen te aciden te ocorri do em 27 de março de 2003 no rio Pomba, um aflu en te do rio Pa raíba do Sul, localizado a sudes te de Minas Gerais. Na ocasião, um enorme derramamento da ordem de 1,4 milhões de metros cúbicos de efluentes industriais, ocasionado pelo rompimento de uma barragem que acumulava resíduos tóxicos produzidos pela fábrica de papel Cataguases, com- 
prometeu seriamen te a biodiversidade do rio Pomba e parte do Paraíba do Sul, até a sua foz (O Globo, 2004).

$\mathrm{O}$ aciden te causou um impacto ambiental de proporções incalculávei s, uma vez que os resíduos tóxicos continham dioxinas e furanos, substâncias químicas organocloradas e cancerígenas que se acumularam nos sedimentos de a mbos os rios. Além disso, o ac i den te provoco $\mathrm{u}$ a interrupção da captação, do tratamento e da d i s tri buição de água, dei x a n docerca de 600 mil pessoas e 8 municípios a jusante do aciden te sem água para o consumo. $\mathrm{O}$ acidente também foi responsável por um impacto negativo na econ omia local, uma vez que as atividades pesqueiras, agrícolas e pecuária foram diret a mente atingidas (Al er j, 2003).

Esse acidente expôs a fragilidade dos órgãos públicos de saúde, meio ambien te e defesa civil em apres entar soluções rápidas para o problema. Exceto a interrupção do abastecimen to por vários dias, nos municípios afetados, para evitar que a contaminação alcançasse as estações de tratamen to de água. $\mathrm{O}$ even to ac a bou reforçando a necessidade de uma agenda política baseada em princípios preventivos ou precaucionários, que estimulem ações e intervenções an tecipatórias na bacia hidrográfica. Também ficou evidente o distanciamen to e a dificuldade dos diferen tes setores que atuam diretamen te com a questão hídrica em trabalhar de forma coopera tiva. As respostas ao acidente se configuraram em ações isoladas, de scoorden adas e aparentemente lentas, no sentido de se apres entar de forma ad hoc um plano de emergência. Isto pôde ser constatado em uma reunião ocorrida no dia 9 de maio de 2003, na sede do Conselho Regi onal de Engenharia e Arqui tetura do Rio de Jan eiro, onde diferentes órgãos e insti tuições se en con traram para avaliar o acidente e tomar medidas relativas ao derramamen to. Através da ata, dis ponível para consulta pública na página el etrônica do Conselho Regional de Engenharia, Arquitetura e Agronomia do Rio Janei ro (Crearj, 2003), foi possível perceber as visões isoladas e fragm en $t$ adas para o problema. Foi verificada também a falta de uma política intersetorial para tratar do acidente. Na verdade, o que se viu foram avaliações setoriais e técnicas, de certa forma distantes da realidade social, política, econômica e cultural da região, que não procurou incorporar de fato os atores não técnicos e lideranças locais diretamen te envo lvidas e atingidas pelo acidente.
Apesar das dificuldades, a vigilância da qu alidade da água possui em seu discurso uma pers pectiva de ação inters etorial, qua n do reco$\mathrm{nh}$ ece a necessidade de integrar diferen tes atores (poder públ i co, setor privado e comunidades) e de descentralização, quando encontra novos espaços de prática sanitária, como os municípios (principal executor das ações), os con selhos gestores delibera tivos (con selhos estadual e municipal de saúde e meio ambiente, comitês de bacia etc.), e as organizações nãogovernamentais.

A gestão participativa para efetivar a vigilância da qualidade da água é um tercei ro de safio. $\mathrm{O}$ en tendimento de pa rticipaçã $o$, dos ge s tores e técnicos que atuam na vigilância e con trole, tem sido o de informar a população e os conselhos de saúde e meio ambiente, sobre a qualidade da água, de forma assimétrica e passiva, por meio de relatórios mensais, que regi stram um determinado estado qualitativo passado, insuficiente para a prevenção de doenças, uma vez que a água contaminada já foi con sumida pela população. Quando ocorre, a tomada de decisão tem sido feita aparen temente de forma centralizada, excluindo o tecido social do processo decisório. Esta centralização pode se acen tuar quando a em presa de abastecimento é do próprio Estado ou município. Neste caso, estabel ecem-se relações de força e poder locais que obstruem a entrada de outros atores no processo, dificultandoa negociação de conflitos e a re s o lução do probl em a.

A participação dos diferen tes atores sociais, como sujeitos ativos na elaboração, formulação, execução e avaliação da qualidade da água para o consumo humano, é fundamental para superar este modelo vigente e hegemônico de vigilância. Um passo fundamental para isto é a disponibilização das informações presentes no Sisagua. Como observou o coordenador do Idec na matéria "Cidades não fiscalizam qualidade da água”, publicada na Folha de S. Paulo, a ...desinform ação impede a conscientização do con sut midor e sua conseqüente cobrança por uma água melhor..

Parti n do do pressu po s to que a vigilância da qualidade da água vem adquirin do contornos epidemiológicos-ambientais, cen trados na avaliação do risco e nos indicadores clássicos de desempenho (Waldman, 1998), como os de utilidade, oportunidade, aceitabilidade, simplicidade, flexibilidade, representatividade, sen sibilidade e valor preditivo positivo, propomos uma ampliação desta idéia de mon i toramen to 
de forma a incorporar os diferen tes atores sociais nesse sistema, numa aproximação ao concei to de vigilância da saúde, que, segundo Teixeira et al. (2001), procura organizar as práticas de saúde e o pró prio funcion a m en to do sistema de saúde, a partir da perspectiva da descentralização das ações de saúde, via municipalização; da intersetorialização do processo saúde-doença; da abertura ao debate para a construção de práticas interdisciplinares; da ampliação da participação comunitária, na busca de uma gestão participativa que desloque as tomadas de decisões para uma base mais h orizontal, a partir do trinômio informaçãodecisão-ação, vis a n doao con trole social.

\section{Con clusões}

E mbora o estabel ecim en to de um programa de vigilância da qualidade da água para consumo humano possa ser considerado um avanço, considerando o históri co dessa vigilância no Brasil, existem ainda muitos desafios para serem su perados. Desafios esses que se esten dem muito mais à necessidade de aproximação da proposta do modelo de vigilância em saúde, resultante da Constituição de 1988 e da lei no $8.080 / 90$, do que da proposta de vigilância em saúde centrada na vigilância dos perigos e das exposições dissociadas dos condicionantes e determinantes socioambientais dos problemas de saúde, orientada para a racionalização das ações do Estado em um con tex to de constrangimentos fiscais.

Não é por acaso que este modelo de vigilância se en con tra cen trado mais no mon i toramen to dos agen tes (vigilância do perigo) que podem com prometer a potabilidade da água, do que da incorporação de condicionantes e determinantes dos probl emas de saúde associados à degradação socioambiental dos recursos hídricos. Assim, além da simples geração, análise e disseminação das informações, o que mesmo na pers pectiva atual só é parcialmen te realizado, há de se ampliar o modelo atual e, por cons eguinte, a pers pectiva de geração e análise de dados, para a construção de informações que possam fortalecer a consciência sanitária e a participação da população, bem como responder às exigências de consolidação do SUS com ênfase na municipalização e na orientação e planeja men to estratégico em saúde.

Quanto ao processo de descentralização, pode-se afirmar que a vigilância da qualidade da água en con tra re s p a ldopolítico e institucional na Programação Pactuada In tegrada de Epidemiologia e Controle de Doenças (PPIECD), a qual vem dando suporte aos municípios para a execução das ações de vigilância. Sente-se ainda que é preciso um inve stimen to de maior amplitude, que atinjaatotalidade dos municípios brasileiros. No levantamen to realizado pelo Instituto Brasilei ro de Defesa do Consumidor (Folha de S. Paulo, 2004), em 2003, nos Estados de Santa Catarina, Paraná, Rio de Jan eiro e São Paulo, constataram-se dificuldades no cumprimen to da portaria no 518, no sentido de se implementar um plano próprio de amostragem; de realizar auditorias nas práticas operacionais das concessionárias; em cadastrar as soluções alternativas de abastecimento; em aprovar o plano de amostragem a pre sen tado pelas con cessionárias; em forn ecer in formações da em presa à população; em manter os regi s tros atualizados e sistematizados sobre a qualidade da água; e realizar a avaliação dos riscos à saúde humana. Pode-se também afirmar que essas dificuldades estão associadas às práticas de gestão das verbas públicas no país, no con tex to da de scen tralização das ações do sistema de saúde. A aplicação dos recursos vindos do Ministério da Saúde para viabilizar as ações de vigilância ainda se constitui num en orme desafio, no sentido da capacidade e do interesse dos ge s tores em lidar com esses recursos. Soma-se a isto a dificuldade dos conselhos de saúde em atuar de forma mais técnica na fiscalização desses mesmos recursos. Um outro grande problema é a falta de profissionais técnicos capacitados para dar su porte ao programa. Este, por exemplo, foi um dos problemas apontados por coordenadores ou responsáveis pelas ações de vigilância ambiental em saúde em 88 municípios investigados no Rio de Janeiro, num levantamento realizado no final de 2003 por Freitas (2003).

Em relação às ações intersetoriais e a uma gestão participativa, há muito que se avançar na vigilância daqualidade da água de consumo humano, que deve incorporar de fato os diferen tes atores sociais, na negociação dos conflitos e nos processos decisórios que envo lvem a qualidade da água em seus diferen tes vieses, ou seja, água para o abastecimen to humano, para o setor produtivo e para a pro teção dos eco s sistemas.

As ações intersetoriais constituem-se em con dutas governamentais, que, no entanto, devem ser pensadas e construídas juntamente 
com a sociedade civil organizada em fóruns de discussão de caráter deliberativos. A criação de linguagens e códigos transversais, envolvendo os diferen tes setores (saúde, meio ambiente, educação, planejam en to, cultura, en ergia etc.), a tores (ONGs, associações, sindicatos, conselhos, comitês de bacia) e fatores (poluição, padrões de consumo, clima gl obal etc.) devem ser estimulados de modo a serem su peradas as dificuldades históricas de ações inter e intra s etoriais.

A incorporação de uma perspectiva sistêmica da qualidade da água, nas esferas do controle social e da gestão participativa, pode favorecer posições mais críticas da população diante das seguintes considerações: o sistema de informação se apres enta como fundamental para mapear áreas de vuln erabilidade e apontar tendências, entretanto sua construção e consolidação devem considerar as possibilidades de aciden tes e even tos ex tremos, como períodos de esti a gem e ench entes, na bacia hidrográfica, assim como as limitações tecnológicas e operacionais do sistema de abastecimen to. Além disso, a sistematização dos dados sobre a qualidade da água deve ser compartilhada de fato. As violações das normas de potabilidade devem ser tratadas de forma transparente, entre poder público, em presas e sociedade, de modo que os parâmetros de potabilidade contidos na portaria no 518 possam ser cumpridos efetivamente.

\section{Colaboradores}

MB Freitas propôs o tema des envo lvido, participou da discussão teórica e metodológica do arti go e da el a boração da redação final, assim como do levantamento bibliográfico sobre o tema abordado. CM Freitas participou da sist ematização e discussão da parte relacionada à vi gilância ambi ental em saúde.

\section{Referências bibliográficas}

ABNT (Ass odação Brasileira de Normas Técnicas) 1996. NBR 12.216: No rma técnica pa ra projetos de estações de tratamento de água. ABNT, Rio de Ja n ei ro.

Alerj (Assembléia Legislativa do Estado do Rio de Janeiro) 2003. Avaliação preliminar da contaminação dos rios Pombae Paraíbado Sul por dioxinas e furanos pela indústria Cataguases de papel. Dis ponível em $\langle h t t p: / /$ www.minc.com.br/mandato/meioambi/dossieriopomba.htm $>$. Acessado em 6 de outubro de 2004.

Be zerra NR, Bonini EM, Ol ivei ra MRL, Net to GF, Dantas MHP 2004. Vigilância da qualidade da água para consumo humano no Brasil: a aplicação da portaria MS no 1469/2000 pelo setor saúde. (Cd Rom). I Congresso Interamericano de Saúde Ambiental, Porto Al egre. 
Gidades não fiscalizam qualidade da água. Folha de $S$. Paulo 20049 abr; p. 1.

Costa EA \& Rozen feld S 2000. A con sti tuição da vigilância sanitária no Brasil, pp. 15-40. In S Rozenfeld (org.). Fundamentos da vigilância sanitária. Fiocruz, Rio de Jan eiro.

Crearj (Conselho Regi onal de Engenharia, Arquitetu ra e Agron omia do Rio de Jan ei ro) 2003. Ata da 2a Reunião da Comissão de Avaliação do Aci den teEcoló gico Ocorri do no Norte/Noroeste Fluminense. Disponível em <http://www.crea-rj.org.br $>$. Acessado em 15 de abril de 2004.

EC (Eu rope Com munity) 2003. Scien tific synthesis report drinking water seminar. 27 and 28 october 2003, Brussels, Bel gium. Dis ponível em $<$ http:// eu ropa.eu. int/>. Acessado em 15 de abril de 2004.

Formaggia DME, Perrone MA, Marinho MJF, Souza RMGL 1996. Portaria 36 GM de 19/01/90: necessidade de revisão. Engenharia Sanitária e Ambiental 1(2):5-10.

Freitas MB 2003. Iden tificando as práticas de saúde ambiental nos municípios do Estado do Rio de Janeiro: su bsídios para compreender a formação profissional em vigilância ambiental em saúde. Relatório Pa rcial do Programa de Aperfeiçoamen to para as Escolas Técnicas/EPSJV/Fiocruz. (Mimeo).

Funasa (Fundação Nacional de Saúde) 2002. Vigilância a mbiental em saúde. Funasa, Brasília.

In forme Epidemiológi co do SUS (IESUS) 1993. Ações de vigilância epidemiológica e controle de agravos na perspectiva do SUS. Informe Epidemiológico do SUS 2(númeroespecial):77-95.

Jouraviev A 2001. Administración del agua en América Latina y el Caribe en el umbral del siglo XX I. Naciones Unidas/Cepis/Edac, SantiagodeChile.

Levy BS 1996. Editorial: toward a holistic approach to public health su rveillance. American Journal of Public Health 86(5):624-625.
Moraes DS \& Jordão BQ 2002. Degradação de recursos híd ri cose seus efei tos sobre a saúde hu m a n a. Revista de Saúde Pública 36(3):370-374.

MS(Ministério da Sa ú de) 2000. Port a riano 1.469. Aprova o padrão de potabilidade da água de consumo humano e dá outras providências. Diário Oficial da União 2000, $29 \mathrm{dez}$.

MS (Ministério da Saúde) 2003. I Seminário Nacional de Saúde e Ambien tecom Controle Social: 16, 17 e 18 de junho de 2003. Mnistério da Saúde, Brasília.

Sherman GJ \& Mathias RG 1996. Editorial: surveillance support for population health decision making. Canadian Journal of Pu blic Health 87(4):221-222.

Tei xeira C 2001. O futuro da prevenção. Casa da Quali$\mathrm{d}$ ade Editora, Sa lvador.

Thacker SB, S troup DF, Pa rrish RG \& An ders on HA 1996. Surveillance in environ mental public health: issues, systems, and sources. American Journal of Public Health 86(5):633-638.

Us epa (United States Envi ronmental Pro tection Agency) 1999. 25 ye ars of the safe drinking water act: history and trends. Dis ponível em $<\mathrm{http} / / / \mathrm{www}$.epa.gov/safe water/consumer/trendrpt.pdf $>$. Acessado em 15 de a bril de 2004

Vazamento envenena água de várias cidades. O Globo 2004 abr 01.

Waldman EA 1998. Usos da vigilância e da monitorização em saúde pública. Informe Epidemiológico do SUS 7(3):7-26.

Wigle D \& Mowat D 1999. Editorial: health surveillance - changing needs, constant function. Canadian Jou $r$ nal of Pu blic Health 90 (3):149-150.

WHO (World Health Organization) 1996. Guidelines for drinking-waterquality, vol. 2. WHO, Geneva.

Artigo apresen $t$ ado em 25/10/2004

Aprovado em 25/11/2004

Versão final apresen tada em 17/06/2005 